\section{Lipase, hepatische}

\section{K. J. Lackner ${ }^{1}$ und D. Peetz ${ }^{2}$}

${ }^{1}$ Institut für Klinische Chemie und Laboratoriumsmedizin, Universitätsmedizin Mainz, Mainz, Deutschland

${ }^{2}$ Institut für Labormedizin, Helios Klinikum Berlin-Buch, Berlin, Deutschland

\section{Synonym(e) EC 3.1.1.3}

Englischer Begriff hepatic lipase

Definition Glykoprotein.

Molmasse Ca. $55 \mathrm{kDa}$.

Beschreibung Die hepatische Lipase (HL) ist eine der beiden hauptsächlichen Triglyzeridlipasen im Plasma. Wie die Lipoproteinlipase ist die HL an das Endothel gebunden und kann dort durch Heparin freigesetzt werden. Im Gegensatz zur Lipoproteinlipase lässt sich die HL nicht durch hohe Salzkonzentrationen $(1 \mathrm{M} \mathrm{NaCl})$ oder Protamin inhibieren. HL benötigt kein Apolipoprotein als Kofaktor. Bevorzugtes Substrat sind wahrscheinlich Triglyzeride aus RemnantPartikeln und IDL. Wie die Lipoproteinlipase hat auch die hepatische Lipase eine Phospholipaseaktivität (A1). Individuen mit genetischen Defekten der HL haben einen relativ unauffälligen Phänotyp mit einer leichten gemischten Hyperlipidämie. Die Aktivität der HL kann ähnlich wie die der Lipoproteinlipase bestimmt werden. Eine Unterscheidung der beiden Aktivitäten kann durch Zugabe inhibierender Antikörper für die HL bzw. Inkubation in Gegenwart von $1 \mathrm{M} \mathrm{NaCl}$ erreicht werden. Die Untersuchung ist derzeit nur von wissenschaftlichem Interesse.

\section{Literatur}

Kobayashi J, Miyashita K, Nakajima K, Mabuchi H (2015) Hepatic lipase: a comprehensive view of its role on plasma lipid and lipoprotein metabolism. J Atheroscler Thromb 22:1001-1011 